\title{
1 Understanding psychophysiological interaction and its relations to beta series correlation
}

3 Xin Di ${ }^{1,2}$, Zhiguo Zhang ${ }^{3,4}$, Bharat B Biswal ${ }^{1,2}$ *

4 1, School of Life Sciences and Technology, University of Electronic Science and Technology of China,

$5 \quad$ Chengdu, China

6 2, Department of Biomedical Engineering, New Jersey Institute of Technology, Newark, NJ, 07029, USA

7 3, School of Biomedical Engineering, Health Science Center, Shenzhen University, Shenzhen, China

8 4, Guangdong Provincial Key Laboratory of Biomedical Measurements and Ultrasound Imaging,

9 Shenzhen, China

10

$11 *$ Corresponding author:

12 Bharat B. Biswal, PhD

13607 Fenster Hall, University Height

14 Newark, NJ, 07102, USA

15 bbiswal@yahoo.com

16 


\section{Abstract}

2 Psychophysiological interaction (PPI) was proposed 20 years ago for study of task modulated

3 connectivity on functional MRI (fMRI) data. A few modifications have since been made, but there

4 remain misunderstandings on the method, as well as on its relations to a similar method named beta series

5 correlation (BSC). Here, we explain what PPI measures and its relations to BSC. We first clarify that the

6 interpretation of a regressor in a general linear model depends on not only itself but also on how other

7 effects are modeled. In terms of PPI, it always reflects differences in connectivity between conditions,

8 when the physiological variable is included as a covariate. Secondly, when there are multiple conditions,

9 we explain how PPI models calculated from direct contrast between conditions could generate identical

10 results as contrasting separate PPIs of each condition (a.k.a. "generalized" PPI). Thirdly, we explicit the

11 deconvolution process that is used for PPI calculation, and how is it related to the trial-by-trial modeling

12 for BSC, and illustrate the relations between PPI and those based upon BSC. In particular, when context

13 sensitive changes in effective connectivity are present, they manifest as changes in correlations of

14 observed trial-by-trial activations or functional connectivity. Therefore, BSC and PPI can detect similar

15 connectivity differences. Lastly, we report empirical analyses using PPI and BSC on fMRI data of an

16 event-related stop signal task to illustrate our points.

17

18 Keywords: beta series, deconvolution, event-related design, functional connectivity, psychophysiological 19 interaction. 


\section{Introduction}

The study of task modulated brain connectivity on functional MRI (fMRI) data is critical for understanding brain functions (Karl J Friston, 2011; Park \& Friston, 2013). Unlike resting-state, where the entire scan period can be treated as a single condition (B. B. Biswal et al., 2010; B. Biswal, Yetkin, Haughton, \& Hyde, 1995; Di \& Biswal, 2014), for task based fMRI, multiple task conditions are typically designed within a scan run. The challenge is to estimate brain connectivity differences between different conditions. In this paper, we mainly focus on two methods that have been developed to study task modulated connectivity for task fMRI data, namely psychophysiological interaction (PPI) (K J Friston et al., 1997) and beta series correlation (BSC) (Rissman, Gazzaley, \& D’Esposito, 2004).

PPI was first proposed by Friston and colleagues based on the interaction term between a physiological variable of a regional time series and a psychological variable of task design in a regression model (K J Friston et al., 1997). According to the definition of effective connectivity (Karl J. Friston, 1994), which refers to the directed effect that one brain region has on another under some model of neuronal coupling, a PPI can be regarded as a condition specific change in effective connectivity, under a simple general linear model (GLM) of interregional coupling. Thereafter, a major update was made to perform deconvolution on the time series from the seed region, so that the interaction term could be calculated at the "neuronal level" rather than at the hemodynamic response level from fMRI signals (Gitelman, Penny, Ashburner, \& Friston, 2003). Later, McLaren and colleagues proposed a "generalized" PPI approach for modeling PPI effects for more than two conditions (McLaren, Ries, Xu, \& Johnson, 2012). They proposed to model each task condition separately with reference to all other conditions and then compare the PPI effects between the conditions of interest, rather than to directly calculate PPI effects between the two conditions. Recently, we found that the interaction between a failure to center the psychological variable and imperfect deconvolution process may lead to spurious PPI effects (Di, Reynolds, \& Biswal, 2017), and the deconvolution may be not a necessary step for PPI analysis on blockdesign data (Di \& Biswal, 2017). 
The BSC method, on the other hand, was primarily proposed for event-related designs (Rissman et al., 2004). By modeling the activations of every trial separately in a GLM, one can estimate a series of

3 beta maps representing the activations of the series of trials. Therefore, connectivity in different task

4 conditions can be calculated and compared in terms of correlations of trial-by-trial beta series variability.

5 According to the distinction of functional connectivity (Karl J. Friston, 1994), which refers to the

6 correlations in measured responses in two areas, a BSC provides a measure of functional connectivity.

7 The relations between the BSC and PPI have not yet been clearly explained. Nevertheless, one study has

8 suggested that BSC method is more suitable for event-related data than PPI (Cisler, Bush, \& Steele, 2014).

9 However, our recent study using a large sample did not support this conclusion (Di \& Biswal, 2019).

In the current paper, we aim to provide an in-depth explanation of the PPI and BSC methods, and explain the relations and differences between these two methods. In order to do so, we need to first clarify why the PPI method always measure connectivity differences between conditions. In addition, we explain the deconvolution process implemented in the calculation of PPI, which can help to understand how PPI can measure the differences between the trial-by-trial dependency in one condition and the moment-to-moment dependency in the remaining time points. Because of this, the PPI differences between conditions and the BSC differences between conditions can, in principle, measure different aspects of task-related modulations of connectivity. We use real fMRI data of an event-related designed task to illustrate our points.

\subsection{Modeling of task main effects}

21 One critical point on understanding modeled effects in a model is that the interpretation of a regressor in a

22 model depends not only on itself but also on how other effects are modeled. To explain this, we start with

23 the modeling of the main effects of task conditions. Assume a simple task design of two conditions A

24 and B, e.g. a flickering checkerboard condition and a fixation baseline. A regression model needs two

25 regressors to represent the two conditions, which can be expressed in two ways. First, one can use the

26 two regressors to represent the specific effect of each condition, i.e. using 1 to represent one condition and 
10 for the other condition (Figure 1A). However, a constant term that represents the overall effect, a.k.a.

2 intercept, is usually added in a regression model. Therefore, we only need to add one more regressor to

3 represent the differential effect between the two conditions (Figure 1B). The two models are

4 mathematically equivalent, because the two regressors in model 1A can be expressed as linear

5 combinations of the two regressors in model 1B, and vice versa. However, because of the differences

6 model strategies, the meanings of the same regressor from the two models (the first regressor from model

$7 \quad 1 \mathrm{~A}$ and the second regressor from model 1B) have changed. In model $1 \mathrm{~A}$, the regressors represent the

8 condition specific effects. In model 1B, however, the second regressor actually represents the differential

9 effect of conditions A and B. This is important regarding the interpretation of the estimated effects of

10 these regressors. Mathematically, model 1B can be expressed as:

$$
y=\beta_{0}+\beta_{1} \cdot x_{P s y c h}+\varepsilon
$$

12 where $x_{P s y c h}$ represents the differential effects between conditions $\mathrm{A}$ and $\mathrm{B}$, i.e. the psychological variable

13 in the context of a fMRI data analysis. $y$ represents the brain signal in a brain region or voxel. $\beta_{0}$ and $\beta_{1}$

14 are parameter estimates that represent the mean effect and differential effect of the two conditions,

15 respectively.

Another important point from equation 1 is that although $x_{P s y c h}$ is usually coded as 1 and 0 for the two conditions, the constant component in $x_{P s y c h}$ is explained by the constant term in equation 1 (see supplementary materials). Thus, whether centering the $x_{P s y c h}$ variable will not affect the effect estimate of $\beta_{1}$, neither the interpretation of $\beta_{1}$. $\beta_{1}$ always represents the differential effect of the two conditions.

\subsection{Functional connectivity and connectivity-task interactions}

23 The term functional connectivity was first defined by Friston (Karl J. Friston, 1994) as temporal

24 correlations between spatially remote brain regions. Assuming that the functional connectivity is the

25 same during the period of scan, e.g. in resting-state, it is straightforward to calculate correlation 
1 coefficients between two brain regions to represent functional connectivity. In a more general regression

2 form, the model can be expressed as:

3

4

$$
y=\beta_{0}+\beta_{1} \cdot x_{\text {physio }}+\varepsilon
$$

where $x_{\text {physio }}$ represents the time series of a seed region. $\beta_{1}$ in this case represents the regression slope, when regressing the activity of the tested voxel on a seed voxel response, i.e., a simple form of effective connectivity.

In most of task fMRI experiments, researchers design different task conditions within a scan run, so that the effect of interest becomes the differences of temporal dependencies between the conditions. We can combine equations 1 and 2 to include both the time series of a seed region (the physiological variable) and the psychological variable into a regression model. Most importantly, the interaction term of the psychological and physiological variables can also be included. For the simplest scenario of only one psychological variable (two conditions), the psychophysiological interaction model can be expressed as:

$$
y=\beta_{0}+\beta_{1} \cdot x_{P s y c h}+\beta_{2} \cdot x_{P h y s i o}+\beta_{3} \cdot x_{P s y c h} \cdot x_{P h y s i o}+\varepsilon
$$

Equation 3 can be illustrated figuratively in Figure 1D. Combine the two terms with $x_{\text {Physio }}$, equation 3 can be expressed as:

$$
y=\beta_{0}+\beta_{1} \cdot x_{P s y c h}+\left(\beta_{2}+\beta_{3} \cdot x_{P s y c h}\right) \cdot x_{P h y s i o}+\mathcal{E}
$$

So that the relationship between the seed region $x_{P h y s i o}$ and test region $y$ is: $\beta_{2}+\beta_{3} \cdot x_{P s y c h}$, which is a linear function of $x_{P s y c h}$. Therefore, a significant $\beta_{3}$ represent significant task modulations on effective connectivity.

Similar to the interpretation of task main effects, the interpretation of the PPI effect depends not only on the PPI itself, but also the on other regressors in the model. In equation 3 the main effect of time series $x_{\text {Physio }}$ is included. We can think about the time series main effect $x_{\text {Physio }}$ and interaction effect $x_{\text {Physio }}$ - $x_{P s y c h}$ as the second order counterparts of the constant effect and main effect of $x_{P s y c h}$ in equation 1. Here, 
1 adding this time series main effect affects the interpretation of the interaction term. Because the overall

2 relationship with the seed time series has been explained by the main effect, the interaction term measures

3 the differences of the relationships between the two conditions. We note that if $x_{\text {Physio }}$ main effect was not

4 added, the interaction term could actually be calculated with each condition separately (Figure 1C). Then

5 the third and fourth columns in Figure 1C can represent condition specific connectivity effects. Again,

6 the same interaction terms from the two models (regressor 3 in model $1 \mathrm{C}$ and regressor 4 in model 1D)

7 represent different effects.

8 In addition, because the main effects of $x_{P h y s i o}$ and $x_{P s y c h}$ are both added in the interaction model

9 (equation 3), the interpretation of the interaction term should refer to the demeaned version of the two

10 variables. Because the $x_{P s y c h}$ is usually coded as 0 and 1 for the two conditions, the demeaned version of

$11 x_{P s y c h}$ will be -0.5 and 0.5 instead. This will make the interaction term look very different (column 4 in

12 Figure 1E compared with that in Figure 1D). However, the estimated interaction effect will be identical,

13 because the difference between the two interaction terms is the physiological main effect, which has been

14 taken into account in the model (For real fMRI data, however, the centering matters because the main

15 physiological main effect interacts with the deconvolution process to produce spurious PPI effects. See

16 Di, Reynolds, \& Biswal (2017) for more details).

17 To better illustrate the meaning of PPI effect, we plot the PPI effect against the original time

18 series $x_{\text {Physio }}$ (Figure 2). PPI can be represented as a projection of the seed time series, so that the PPI

19 represents different relationships with the seed region in different task conditions. When the

20 psychological variable is coded as 1 and 0 for the two conditions, the PPI represents a perfect relationship

21 with the seed time series in the " 1 " condition and a smaller effect in the " 0 " condition, which is reflected

22 as a horizontal line in Figure 2D. When the mean of the psychological variable is removed before

23 calculating the interaction term, the projection rotates clockwise compared with the non-centered version

24 (Figure 2G). However, what is reflected in the two projections are the same, which is the difference

25 between the two conditions. In real cases, there may be positive connectivity in condition A and no 
1 connectivity in condition B, or there may be no connectivity in condition A but negative connectivity in

2 condition B. In both cases, PPI can capture the differential connectivity effects between A and B.

[Insert Figure 2 about here]

\subsection{More than two conditions}

6 If we now consider multiple conditions or experimental factors, there is an opportunity to evaluate the

7 differences in the PPI that is induced by one psychological factor, relative to another. This becomes a

8 difference in PPIs that, mathematically, can be thought of as the interaction between the two

9 psychological factors in mediating the interaction with the physiological fluctuations. Technically, this is

10 a three-way interaction sometimes referred to as a psycho-psychophysiological interaction (PPPI) (Rowe,

11 Stephan, Friston, Frackowiak, \& Passingham, 2005). For example, if two sorts of face stimuli were

12 presented (e.g., angry versus neutral) a transient increase in coupling from the amygdala to the fusiform

13 area might be greater under the angry faces (relative to no faces), compared to the neutral faces (relative

14 to no faces). In other words, the condition specific change in effective connectivity itself depends upon

15 whether the stimuli were angry or neutral.

When considering modeling $n$ number of conditions, the same $n$ regressors are needed. Because

17 there is always a constant term in the regression model, we actually need $n-1$ additional regressors. This

18 is convenient for most task fMRI studies, because there is usually an implicit baseline condition, e.g. a

19 resting or fixation condition. For event-related design, it is even difficult to explicitly define the baseline

20 condition. Therefore, we can include all the experimental conditions, and leave the baseline condition out

21 of the model. Because of the inclusion of the constant term, we should always keep in mind that the

22 regressors included in the model represent the differences of between the modeled condition with respect

23 to all the other conditions, rather than the specific effect of a condition.

24 Here assume a task design with conditions A and B together with a baseline condition R. The

25 effect of interest is the differences between conditions A and B. A natural way to model the three

26 conditions is to use two regressors to represent A and B, separately (Figure 3B and 3D). We could then 
1 calculate the interaction terms of the two psychological regressors with the seed time series, respectively.

2 The two interaction terms represent the connectivity differences effects of $A-(B+R)$ and $B-(A+R)$,

3 respectively. A contrast between these two, i.e. $[A-(B+R)]-[B-(A+R)]=2 \times(A-B)$, can then be

4 used to examine the differences between A and B. This strategy is usually referred to as "generalized"

5 PPI (McLaren et al., 2012). Alternatively, one can directly contrast A with B to define a new

6 psychological variable. It can be achieved in SPM by defining contrast value 1 to condition A, and -1 to

7 condition B (Figure 3F). However, don't forget the third condition R, which will be implicitly left as 0.

8 Simply doing this is problematic, because it assumes that the relationship in the R condition is somehow

9 between what are in the A and B conditions (Figure 3G). Because there are three conditions in total, we

10 have to use two variables to model the differences among the three conditions. In this case, we could

11 include one more psychological variable to represent the differential effect between the mean effect of A

12 and $\mathrm{B}$ and the effect of $\mathrm{R}$ (Figure $3 \mathrm{H}$ ). The interaction term of this psychological variable with the seed

13 time series can effectively remove the differential effects of relationships between conditions A/B and

14 condition R (Figure 3I). Therefore, if we include the PPI terms of the psychological variables $3 \mathrm{~F}$ and $3 \mathrm{H}$

15 in the model, the effect corresponding to $3 \mathrm{~F}$ will be equivalent to the differential effects of the PPIs

16 corresponding to 3B and 3D. In the original paper of McLaren, it has been shown that the "generalized"

17 PPI approach performed better than the contrast PPI. It is probably because of the neglect of the $\mathrm{R}$

18 condition. If the psychological variables are modeled correctly, the two methods should provide the same 19 results.

[Insert Figure 3 about here]

\subsection{Block design and event-related design}

23 So far we have divided the observations of different task conditions into different groups regardless of the

24 orders of the observations. For fMRI, the task conditions need to be designed carefully to accommodate

25 the properties of hemodynamic responses following neural activity changes due to task designs. There

26 are usually two types of designs, block and event-related designs. For the block design, a task condition 
1 is broken into separate short blocks, and the blocks are repeated for several times within a scan run. In

2 block designs, the psychological factor is usually thought of as a context (e.g., attentional set). In this

3 setting, the PPI can be interpreted in terms of the difference in the regression slopes when regressing

4 activity in one area on the other, under the two experimental contexts. If one interprets the regression

5 slope has a simple measure of effective connectivity, then the PPI corresponds to the change in effective

6 connectivity, given the experimental context or psychological factor.

$7 \quad$ For event-related design, each trial is a unit to evoke hemodynamic responses. The temporal

8 distance between trials should be designed carefully, so that the hemodynamic response for each trial

9 could be effectively separated (Dale, 1999; K J Friston et al., 1998). The psychological variable for

10 event-related design is modeled as a series of impulse function at the onset of the trials with remaining

11 time points as 0. Mathematically, exactly the same interpretation holds for event related designs;

12 however, here, the event is very short lived and cannot be interpreted as a context. A PPI in event related

13 designs reflects a transient increase (or decrease) in the effective connectivity (in a trial-by-trial manner)

14 between two brain areas that can be attributed to the stimulus for action associated with the event in

15 question. For example, a visual stimulus may transiently enhance the coupling between the amygdala and

16 fusiform area, in relation to baseline coupling in the absence of a stimulus.

\section{$18 \quad$ 1.5. Convolution and deconvolution}

19 One important aspect of fMRI is the asynchrony between the (hypothetical) neuronal activity and the 20 observed blood-oxygen-level dependent (BOLD) signals. A trial or short event elicits transient neural

21 activity that is typically treated as an impulse function, and further gives rise to a delayed hemodynamic

22 response, which is usually called hemodynamic response function (HRF) (Figure 4A). If there are

23 designed trials or hypothetical neural activity, the expected BOLD responses can be calculated as a

24 convolution of the design or neural activity time series with the HRF. Because the fMRI data are discrete

25 signals, the convolution can be converted into a multiplication of the neuronal signal with a convolution 
1 matrix corresponding to the HRF. Using $z$ to represent variables at the neuronal level, and $x$ to represent

2 variables at the BOLD level, the convolution can be expressed as:

$$
x=z * h=H \cdot z
$$

$4 \quad$ where * represents the convolution process, $\cdot$ represents matrix multiplication, $h$ is the HRF, and $H$

5 represents the matrix form of $h$. Each column of $H$ represents a HRF with a different start point (Figure

$64 \mathrm{~B})$. Therefore, the multiplication of a neural time series $H$ with $z$ can be understood as a summation of

7 the hemodynamic responses of $z$ at every time point.

[Insert Figure 4 about here]

For fMRI, we typically hypothesize that an experimental manipulation will evoke immediate neural response (relative to the time scale of BOLD responses). The expected BOLD responses to the experimental manipulations could then be represented as the convolution of the psychological variable $z_{\text {Psych }}$ (a box-car function or a series of impulse functions) with the HRF. Thus, the BOLD level prediction variable $x_{P s y c h}$ can be calculated from $z_{P s y c h}$ as the following:

4

$$
x_{P s y c h}=z_{P s y c h} * h
$$

On the other hand, through fMRI we have a time series of a region $x_{\text {Physio, }}$, which is already at the BOLD level. Therefore, we can directly calculate the interaction term by multiplying $x_{\text {Physio }}$ with $x_{P s y c h}$.

$$
x_{P P I}^{1}=x_{P s y c h} \cdot x_{P h y s i o}
$$

This is how PPI was calculated when the method was originally proposed (K J Friston et al., 1997). The limitation of this approach is that it calculates the interaction at the BOLD level, but the real interaction would happen at the "neuronal" level.

Given the BOLD level time series $x$, we can perform the inverse of convolution, i.e. deconvolution, to recover the time series $z$ at the neuronal level from equation 5. However, the $H$ matrix is a square matrix, so that deconvolution cannot be simply solved by inverting the $H$ matrix. In addition, for practical deconvolution problem like the fMRI signals, there are always noises in the recorded signals 
1 that need to be taken into account. Therefore, the deconvolution problem has to solve the following

2 model with a noise component $\varepsilon$.

$$
x=H \cdot z+\varepsilon
$$

4 Because $H$ cannot be directly inverted, some computational methods like regularization are needed to

5 reliably obtain $z$. In SPM, it additionally substitutes $z$ with Discrete Cosine Series, so that the estimation

6 of temporal time series was transformed into frequency domain (Gitelman et al., 2003). And the

7 regularization is applied to specific frequency components.

8 A seed time series $x_{\text {Physio }}$ can be deconvolved to the neuronal level time series $z_{\text {Physio }}$ and

9 multiplied with the neuronal level psychological variable. The interaction term can then be convolved 10 back into BOLD level:

$$
x_{P P I}^{2}=\left(z_{P s y c h} \cdot z_{P h y s i o}\right) * h
$$

$12 x_{P P I}^{1}$ and $x_{P P I}^{2}$ are not mathematically equivalent. The later one is more appropriate to describe neural 13 interactions. However, empirically, the PPI terms calculated with the two ways could be very similar for

14 block designs (Di \& Biswal, 2017). In addition, deconvolution is an ill-posed problem, and relies on 15 sophisticated computational techniques, which may not work well in some circumstances. Therefore, it 16 has been suggested that at least for block design, deconvolution may not be necessary (Di \& Biswal, 2017;

17 O'Reilly, Woolrich, Behrens, Smith, \& Johansen-Berg, 2012). The deconvolution approach may still be 18 important and necessary for event-related design.

\subsection{Beta series correlations}

21 BSC is based on a simple idea of calculating correlations of trial-by-trial variability of brain activations.

22 Instead of modeling different task conditions, BSC models every trial's activation to obtain a beta map for

23 each trial. For each trial, an impulse function at the trial onset is defined and convolved with HRF.

24 Therefore, a GLM for BSC analysis has the same number of regressors as the number of trials plus a

25 constant term. The model can be expressed as the following: 


$$
y=\beta_{0}+\beta_{1} \cdot x_{1}+\beta_{2} \cdot x_{2}+\cdots+\beta_{1} \cdot x_{1}+\beta_{n} \cdot x_{n}+\varepsilon
$$

where $n$ represents the number of trials, and $x_{n}$ represents the modeled response of the trial $n$. The model can be expressed in a matrix form:

$$
y=X \cdot \beta+\varepsilon
$$

where $\beta$ represents a vector of $\beta$ s corresponding to the activations of different trials (plus a $\beta_{0}$ for the constant term). Figure 5 shows examples of the design matrix $X$. One can then calculate cross-trial correlations of the beta values between regions to represent functional connectivity. Since there are usually more than one experimental condition, the beta series can be retrospectively grouped into different conditions, and the beta series correlations can be compared between the conditions.

[Insert Figure 5 about here]

The hemodynamic response typically reaches the peak at $6 \mathrm{~s}$ after trial onset and returns back to the baseline after about $15 \mathrm{~s}$. To avoid overlaps of hemodynamic responses between trials, some eventrelated experiments use slow designs with intertrial interval usually greater than $10 \mathrm{~s}$. Figure 5A demonstrates a beta series GLM for a slow event related design from a Flanker task (Kelly, Uddin, Biswal, Castellanos, \& Milham, 2008). Considering a typical sampling time of $2 \mathrm{~s}$ for fMRI, the design matrix of Figure 5A can be reliably inverted (24 trial regressors vs. 146 time points). However, fast event-related design is becoming more standard, because of its efficiency of maximizing experimental contrasts (Dale, 1999; K J Friston et al., 1998). The intertrial interval could be close to the sampling time of fMRI for some designs. Figure 5B demonstrated a beta series GLM for a fast event-related design from a stop signal task (Di \& Biswal, 2019). In this case the mean intertrial interval is $2.5 \mathrm{~s}$. It can be seen that the number of regressors becomes closer to the number of time points (126 trial regressors vs. 182 time points). This matrix cannot be reliably inverted using ordinary least squares (OLS) method, and some sophisticated computational methods may be helpful to resolve the problem, e.g. using regularization or modeling a single trial against all other trials to reduce the number of regressors (Di \& Biswal, 2019; Mumford, Turner, Ashby, \& Poldrack, 2012). 
The beta values in the beta series model typically represent BOLD level activations at each trial. However, in a case when the trials are presented at every time point, the beta series model becomes exactly the same as the convolution matrix in Figure 4B. The beta series model estimates transient neuronal responses specifically at each trial onset, while the deconvolution model estimates neuronal responses at every time point. This suggests a link between the beta series model and deconvolution.

\subsection{The relations between PPI and BSC}

8 As described in previous sections, the BSC method estimates neuronal activity for each trial, and

9 computes trial-by-trial correlations between brain regions. The correlations reflect functional connectivity of a task condition. The PPI, on the other hand, measures connectivity differences as coded by a psychological variable. Therefore, a beta series correlation in one condition is not comparable to a simple PPI effect. However, for task fMRI what is usually of interest are the differences between task conditions. Considering the same task design with experimental conditions A, B, and a baseline R, as has been explained, both contrast PPI and "generalized" PPI can measure effective connectivity effectively a change in regression slope or parameter of a model of effective connectivity. In contrast, differences in BSCs are changes in the statistical coupling or functional connectivity. This is important because one can have a change in a correlation without a change in effective connectivity. This can occur when the random noises in one experimental condition differ from the other. results of PPI and BSC on real fMRI data may not be identical. Several factors may contribute to the

24 differences. The first is the different approaches to deconvolution. The deconvolution method

25 implemented in SPM uses Discrete Cosine Series to convert the temporal domain signal into frequency

26 domain, and then applies regularization on the frequency domain to suppress high frequency components 
1 in the signals. For BSC method, if it is a slow event-related design, single-trial activations can be directly

2 estimated from the GLM. For a fast event-related design, some regularization methods or complicated

3 modeling strategies are required to estimate the beta series (Mumford et al., 2012). The efficiency and

4 reliability of these mentioned methods are difficult to determine and compare. And it may depend on the

5 intertrial intervals of a design (Abdulrahman \& Henson, 2016; Mumford, Davis, \& Poldrack, 2014;

6 Visser et al., 2016), or different brain regions due to different amount of HRF variability (Handwerker,

7 Ollinger, \& D’Esposito, 2004).

8 Another difference may be the different statistical measures of connectivity. By using a

9 regression model PPI essentially measures the differences in slope between conditions. On the other hand,

10 BSC typically uses correlation coefficients. It is still largely unknown how the variability of BOLD

11 signals changes in different task conditions (Duff, Makin, Cottaar, Smith, \& Woolrich, 2018), which may

12 influence PPI measures. For BSC, one can choose different measures of connectivity, e.g. Pearson's

13 product-moment correlation, Spearman's rank correlation, covariance, or even similar beta series-by-task

14 interaction as PPI under a regression model to estimate connectivity differences. However, it is still an

15 open question about which method is optimal for the purpose of connectivity estimations.

\section{1.8. An empirical demonstration}

18 In summary, PPI analyses allow one to test for condition specific differences in (linear) effective connectivity between two areas. Furthermore, by comparing PPIs associated with different conditions one can test for high order context sensitive changes in coupling. An obvious but interesting hypothesis

21 here is that the presence of such high order interactions might manifest as changes in functional

22 connectivity between two experimental conditions. One can access these changes in functional

23 connectivity by leveraging the trial-by-trial variability in evoked responses with a BSC. One can then

24 simply look at the differences in these correlations (i.e., functional connectivity) to see if they identify the 25 same pairwise connections detected by "generalized" PPI analysis. We will demonstrate this using 26 empirical data in an event related setting. 
We analyzed an fMRI dataset with a fast event-related designed stop signal task. There were two experimental conditions (Go trials and Stop trials) in addition to an implicit baseline. The connectivity

3 differences between the Stop and Go conditions have been reported previously (Di \& Biswal, 2019).

4 Here, we report connectivity measures with PPI and BSC for simple conditions and condition differences.

5 The PPI and BSC results are also compared with resting-state functional connectivity to better illustrate

6 their relations to each other. Secondly, we will show that the "generalized" PPI approach and direct

7 contrast PPI approach provide identical measures of effective connectivity differences between conditions.

8 Lastly, we will compare different correlation measures for BSC analysis, i.e. Pearson's correlation,

9 Spearman's correlation, covariance, and beta series-by-task interaction.

\section{Materials and methods}

\subsection{Dataset and designs}

13 In our previous paper, we have reported the PPI and BSC results of connectivity differences between the

14 Stop and Go conditions (Di \& Biswal, 2019). In the current paper, we used the same data to illustrate

15 how different PPI models could give rise to the same results and how the PPI and BSC methods can be

16 similar or different. This dataset was obtained from the OpenfMRI database (accession \#: ds000030).

17 Only healthy subjects' data were included in the current analysis. After removing subjects due to large

18 head motion, a total of 114 subjects were included in the current analysis ( 52 females). The mean age of

19 the subjects was 31.1 years (range from 21 to 50 years). In the stop signal task, the subjects have to

20 indicate the direction (left or right) of an arrow presented in the center of the screen. For one fourth of the

21 trials, a $500 \mathrm{~Hz}$ tone was played shortly after the arrow, which signaled the subjects to withhold their

22 response. In a single fMRI run, there were 128 trials in total in total, with 96 Go trials and 32 Stop trials.

23 The task used a fast event-related design, with a mean intertrial interval of $2.5 \mathrm{~s}$ (range from $2 \mathrm{~s}$ to $5.5 \mathrm{~s}$ ).

24 For a subset of 103 subjects, we also analyzed their resting-state fMRI data. The exclusion of additional

25 subjects were due to large head motions in either the resting-state run or other task runs that were not

26 included in this paper. 
The fMRI data were collected using a T2*-weighted echoplanar imaging (EPI) sequence with the

2 following parameters: $\mathrm{TR}=2000 \mathrm{~ms}, \mathrm{TE}=30 \mathrm{~ms}, \mathrm{FA}=90 \mathrm{deg}$, matrix $64 \times 64$, FOV $=192 \mathrm{~mm}$; slice

3 thickness $=4 \mathrm{~mm}$, slice number $=34.184 \mathrm{fMRI}$ images were acquired for each subject for the stop signal

4 task, and 152 images were acquired for the resting-state run. The T1 weighted structural images were

5 collected using the following parameters: $\mathrm{TR}=1900 \mathrm{~ms}, \mathrm{TE}=2.26 \mathrm{~ms}, \mathrm{FOV}=250 \mathrm{~mm}$, matrix $=256 \times$

6256 , sagittal plane, slice thickness $=1 \mathrm{~mm}$, slice number $=176$. More information about the data can be

7 found in (Poldrack et al., 2016).

8

\subsection{FMRI preprocessing}

The fMRI image processing and analysis were performed using SPM12 (v6685)

\section{(http://www.fil.ion.ucl.ac.uk/spm/) and scripts in MATLAB R2013b environment}

(https://www.mathworks.com/). The anatomical image for each subject was first segmented, and normalized to standard MNI (Montreal Neurological Institute) space. The first two functional images were discarded, and the remaining images were realigned to the first image, and coregistered to the subject's own anatomical image. The functional images were then transformed into MNI space by using the deformation images derived from the segmentation step, and were spatially smoothed using a $8 \mathrm{~mm}$ FWHM (full width at half maximum) Gaussian kernel.

\subsection{PPI analysis}

The first step of PPI analysis is to build a voxel-wise GLM for task activations. In the current analysis, the Go and Stop conditions were modeled separately as series of events (trials). In SPM, the durations of events are usually set as 0 to reflect their impulsive nature. But for PPI analysis, the time series are upsampled (16 times by default) after deconvolution. If the duration is set as 0 , then the neuronal level psychological variable only has a time bin of one with duration of TR/16, leaving all other time bins as 0 . This may be problematic when multiplying this psychological variable with the deconvolved seed time series. Considering that the calculated PPI term will be convolved back with HRF, which resembles a 
1 low pass filtering, the effects of trial duration may not be that significant. In the previous analysis, we set

2 the duration to $1.5 \mathrm{~s}$, which represented the actual duration of the trial. We have also shown in the

3 supplementary materials that setting the event duration as 0 produce very similar results as those with 1.5

$4 \mathrm{~s}$ duration. In the results section, we report results with $1.5 \mathrm{~s}$ duration. In addition to the two event-

5 related task regressors, 24 head motion regressors and one constant regressor were also included in the

6 GLM model. After model estimation, the times series from 164 ROIs were extracted. The head motion,

7 constant, and low frequency drift effects were adjusted during the time series extraction. These 164 ROIs

8 were adopted from previous studies (Di \& Biswal, 2019) to represent whole brain coverage. The

9 following connectivity analyses of PPI and BSC were performed on ROI-to-ROI basis.

10 The PPI terms were calculated using the two different approaches. For the "generalized" PPI

11 approach, we used the contrasts [ $\left[\begin{array}{ll}1 & 0\end{array}\right]$ and [ $\left[\begin{array}{ll}0 & 1\end{array}\right]$ to define the two psychological variables representing the

12 Go and Stop conditions, separately. The PPI terms were then calculated using the deconvolution method.

13 The calculated PPI terms were combined together with the original model to form a new GLM for PPI

14 analysis:

$$
y=\beta_{0}+\beta_{1} \cdot x_{G o}+\beta_{2} \cdot x_{S t o p}+\beta_{3} \cdot x_{R O I}+\beta_{4} \cdot x_{P P I: G o}+\beta_{5} \cdot x_{P P I: S t o p}+\varepsilon
$$

This model included one constant term, two regressors of task activations, one regressor of the ROI time

series, and two regressors of PPIs. Here the differential effect of the two PPI terms, $\beta_{5}-\beta_{4}$, represents the higher order task modulated connectivity (i.e. PPPI) between the Stop and Go conditions. Because the dependent variable $y$ is also a ROI time series, where the head motion effects have already been removed, the head motion regressors were no longer included in the PPI models.

22 and Go conditions using the contrasts [-1 1] and [1/2 1/2], respectively. After PPI calculation using the deconvolution method, the GLM for the contrast PPI analysis was defined as follow:

$$
y=\beta_{0}+\beta_{1} \cdot x_{G o+S t o p}+\beta_{2} \cdot x_{S t o p-G o}+\beta_{3} \cdot x_{R O I}+\beta_{4} \cdot x_{P P I: G o+S t o p}+\beta_{5} \cdot x_{P P I: S t o p-G o}+\varepsilon
$$


1 The estimates of $\beta_{5}$ were used for group level analysis to present connectivity differences between the

2 Stop and Go conditions.

For each subject, the PPI models were built for each ROI, and were fitted to all other ROIs. The

4 beta estimates or contrasts of interest were calculated between each pair of ROI, which yielded a 164 by

5164 matrix for each effect. The matrices were transposed and averaged with the original matrices, which

6 yielded symmetrical matrices. This procedure ensures that the PPI matrices can be compared to the

7 (symmetrical) undirected functional connectivity matrices furnished by BSC. One sample t test was

8 performed on each element of the matrix for an effect of interest. False discovery rate (FDR) correction

9 was used at $\mathrm{p}<0.05$ to identify statistical significant effects in a total of 13,366 effects $(164 x(164-1) /$

$102)$.

\subsection{Beta series analysis}

13 As has been shown in our previous paper (Di \& Biswal, 2019), modeling all trials together in a single model could not work for the beta series analysis. Therefore, we only reported the results from the singletrial-versus-other-trials method (Mumford et al., 2012). We first built a GLM for each trial, where the first regressor represented the activation of the specific trial and the second regressor represented the

17 activations of all the remaining trials. The 24 head motion parameters were also included in the GLMs as covariates. The duration of events was set as 0 . After model estimation, beta values of each ROI were extracted for each trial. The beta series of each ROI were sorted into the two conditions, and the functional connectivity measures across the 164 ROIs were calculated. In our previous work, we used

21 Spearman's rank coefficients to avoid Gaussian distribution assumption of the beta series or spurious

22 correlations due to outliers. In the current analysis, we also calculated Pearson's correlation coefficients,

23 covariance, and beta series-by-task interaction to examine whether these two measures may give more

24 reliable estimates of connectivity. The whole beta series (Go and Stop together) of a ROI were first z

25 transformed, and then the correlation coefficients and covariance matrices were calculated for each

26 subject. The Pearson's and Spearman's correlation matrices were transformed into Fisher's z matrices. 
1 Similar to PPI, we calculated beta series-by-task interaction for each ROI, and built a GLM including the

2 raw beta series main effect, task main effect, their interaction, and a constant term. The GLM was used to

3 predict the beta series of every other ROI. The $\beta s$ corresponding to the interaction term were used to

4 represent task modulated connectivity. The $\beta$ matrices were transposed and averaged with the original

5 matrices, resulting in symmetrical matrices.

$6 \quad$ For a single condition, the mean of Fisher's z values or covariance values were averaged across

7 subjects. Paired t tests were also performed to compare the differences between the two conditions at

8 every element of the matrix. For the beta series-by-task interaction analysis, one sample $t$ test was used in

9 the group level analysis. A FDR correction at $\mathrm{p}<0.05$ was used for all the measures to identify statistical 10 significant effects.

13 A voxel-wise GLM was first built for each subject, which included 24 head motion regressors and on

14 constant term. After model estimation, the times series from the 164 ROIs were extracted, adjusting for

15 the head motion, constant, and low frequency drift effects. For each subject, a Pearson's correlation coefficient matrix was calculated across the 164 ROIs. The matrices were transformed into Fisher's z

17 matrices, and averaged across subjects.

\section{Results}

\section{1. “Generalized” PPI vs. BSC}

21 We first show task modulated connectivity matrices calculated from "generalized" PPI and BSC (Figure

22 6). To illustrate overall patterns, the matrices were not thresholded. Consistent with our explanations of

23 the differences between PPI and BSC, the simple PPI effects of a condition look very different from those

24 calculated from BSC. For the Go condition, the PPI matrix (shown in Figure 6A, corresponding to $\beta_{4}$ in

25 equation 12) had increased connectivity between visual and sensorimoter regions and between cerebellar

26 and sensorimotor regions, and decreased connectivity within visual areas compared with the other 
1 conditions. In contrast, the BSC matrix (Figure 6D) of the Go condition showed square like high

2 correlations along the diagonal, which represent higher functional connectivity within each functional

3 modules. The correlation between the PPI and BSC matrices of the Go condition was only 0.17 (Figure

$46 \mathrm{G}$ ). Similarly, the PPI (Figure 6B, corresponding to $\beta_{5}$ in equation 12) and BSC (Figure 6E) matrices of

5 the Stop condition also looked different, with a small correlation of -0.20 (Figure 6H). However, despite

6 the differences in single conditions, the differential effects between the Stop and Go conditions were

7 similar between the PPI (Figure 6C) and BSC (Figure 6F) methods. The correlation between the two

8 matrices was 0.73 (Figure 6I), which has been reported previously (Di \& Biswal, 2019).

9

[Insert Figure 6 about here]

The BSC matrices of both the Stop and Go conditions looked very similar to each other, and were indeed similar to resting-state correlations. To confirm this, we analyzed the resting-state fMRI data from a subset of 103 subjects, and calculated resting-state functional connectivity matrix (Figure 7A). The correlations between the resting-state connectivity and the BSC matrices of the Go and Stop conditions were 0.91 (Figure 7B) and 0.92 (Figure 7C), respectively.

[Insert Figure 7 about here]

\section{2. “Generalized" PPI vs. contrast PPI}

We also performed PPI analysis using the contrast PPI approach, i.e. modeling the mean and differential effects of the Go and Stop conditions, respectively. The mean PPI effects of the Go and Stop conditions (Figure 8A, corresponding to $\beta_{4}$ in equation 13) looked similar to the specific effects of each condition from the "generalized" PPI approach (Figure 6A and 6B). Most interestingly, consistent with our explanation, the differential effects of the Stop and Go conditions (Figure 8B, corresponding to $\beta_{5}$ in equation 13) turned out to be identical to the contrast of Stop and Go PPI effects from the "generalized" PPI model (Figure 6C). This can be confirmed by showing the scatter plot between the two matrices (Figure 8C), which demonstrated as a straight line. It should be noted that the effects of the "generalized" PPI (contrast values) were as two times as the effects of the contrast PPI. This has been explained in 
1 section 1.3 that the contrast between two "generalized" PPI effects A and B represent the effect of 2 x (A $2-\mathrm{B})$.

[Insert Figure 8 about here]

The mean effects of the Go and Stop conditions, which reflect task modulated connectivity related to general task execution, have not been reported previously. We performed one sample t test on every element of the matrix. Statistical significant effects were thresholded at $\mathrm{p}<0.05$ (FDR corrected) and visualized using BrainNet Viewer (Xia, Wang, \& He, 2013) (Figure 9). It clearly shows that there was reduced effective connectivity within the visual areas, and increased connectivity mainly between visual regions and sensorimotor regions and between visual regions and other brain regions such as cingulo-opercular regions.

[Insert Figure 9 about here]

\subsection{Different BSC measures}

Lastly, for the contrast of Stop vs. Go where the PPI and BSC methods yielded similar results, we compared different correlation measures for the BSC analysis (Figure 10). When comparing the four measures of Spearman's correlation, Pearson's correlation, covariance, and beta series-task interaction, Pearson's correlation produced the largest number of significant effects, while covariance only showed one significant positive effect and one significant negative effect. However, even the results from Pearson's correlation showed less significant results than the PPI model.

[Insert Figure 10 about here]

\section{Discussion}

In the current paper, we have explained that because the inclusion of the physiological variable in the PPI model, a PPI effect always represents the differences of regression parameters between conditions. In an event-related setting, a PPI reflects effective connectivity differences between those elicited by transient events and those during the baseline condition. In contrast, BSC measures functional correlations elicited 
1 by series of transient events in a specific task condition. However, when comparing between conditions,

2 BSC should in principle yield similar estimates as PPI differences (i.e. PPPI effects). The results of PPI

3 and BSC analyses on fMRI data of an event-related designed stop signal task agree with our theoretical

4 explanation of the two methods. Firstly, the BSCs of the Go and Stop conditions were very similar to

5 each other, and were also highly correlated with the functional connectivity in resting-state. In contrast,

6 the PPIs of the Go and Stop condition from the "generalized" PPI approach tuned out to be very different

7 from those from the BSC analysis. Secondly, when contrasting the Stop and Go conditions, the PPI

8 matrices and BSC matrices were very similar, which reflected the connectivity differences between the

9 conditions. Lastly, consistent with our mathematical explanation, direct contrast PPI showed exactly the

10 same results as "generalized" PPI when the conditions were modeled properly.

As explained in the introduction, the correlations of trial-by-trial variability measured by BSC

12 reflect the absolute level of functional connectivity in a condition. Interestingly, we found that the BSCs

13 for the Go and Stop conditions were very similar to each other, and were also similar to what we typically

14 observed in resting-state. This is consistent with the observation that the moment-to-moment correlations

15 in many different task conditions are very similar (Cole, Bassett, Power, Braver, \& Petersen, 2014). This

16 suggests that the absolute correlations between brain regions in any task conditions are highly contributed

17 by the correlations of spontaneous neural activity (B. Biswal et al., 1995), or other common factors that

18 could give rise to high correlations, e.g. common anatomical connectivity (Honey et al., 2009), common

19 neurovascular responses (Hillman, 2014; Sivakolundu et al., 2019), physiological noises (Weissenbacher

20 et al., 2009), or head motion (Power, Barnes, Snyder, Schlaggar, \& Petersen, 2012; Van Dijk, Sabuncu, \&

21 Buckner, 2012). Nevertheless, the similarity of absolute correlations in many different task conditions

22 including resting-state makes them lack of interest in terms of understanding brain functions. Practically,

23 it would be more informative and valuable to examine whether connectivity is modulated by a task

24 manipulation than to only look at the absolute value of the connectivity. 
It is noteworthy that in equation $3, \beta_{2}$ represents task independent connectivity between the seed region $x_{P h y s i o}$ and test region $y$, after covarying task dependent connectivity and task activation. Further,

3 given the linear function of the relation between $x_{P h y s i o}$ and $y$ in equation 4 , i.e. $\beta_{2}+\beta_{3} \cdot x_{P s y c h}$, one can also

4 recover functional connectivity between the two in a specific task condition encoded in $x_{P s y c h}$. But one

5 should be cautious that due to centering and deconvolution process, the values in $x_{P s y c h}$ to represent one

6 task condition will no longer be 1 (usually smaller than 1). Simply using $\beta_{2}+\beta_{3}$ will over emphasize task

7 modulated connectivity, and cannot be a good estimate of task specific connectivity.

The PPI matrices of the Go or Stop conditions from the "generalized" PPI approach were very

9 different from the BSC matrices of respective conditions. This is because the PPI effects for one condition reflect connectivity differences between the very condition and the rest of the experiment period. This cannot be achieved by using the BSC method, because the implicit baseline conditions cannot be easily modeled as events. Compared with the implicit baseline, the Go and Stop conditions showed decreased connectivity between visual areas, and increased connectivity between visual areas and sensorimotor areas among other brain regions. The reduced connectivity within the visual areas during task execution compared with baseline is consistent with our previous studies using a set of different tasks (Di et al., 2017) as well as in a simple checkerboard task (Di \& Biswal, 2017). However, in contrast to

17 the reduced functional connectivity between visual and sensorimotor regions in the checkerboard task (Di \& Biswal, 2017), the current results showed increased functional connectivity between the visual and sensorimotor regions. It is not surprising because the stop signal task requires the subjects to response to visual stimuli, therefore yielding increased functional coupling between visual and sensorimotor regions. When directly comparing the differences between the Stop and Go conditions, both PPI and BSC methods showed similar results, which are consistent with our explanations in the introduction. In

23 addition to Spearman's correlation used in our previous paper (Di \& Biswal, 2019), we further compared

24 BSC differences using Pearson's correlation, covariance, and beta series-by-task interaction in the current 25 analysis. Pearson's correlation did yield more statistical significant effects than Spearman's correlation, 26 however, all the four measures showed less number of significant effects than the PPI analysis. Therefore, 
1 in terms of statistical sensitivity, there is no support for the claim that BSC is more suitable for event-

2 related designed data (Cisler et al., 2014).

In this paper, we have explained the deconvolution process for the BOLD signals, which can help

4 to understand the relations between PPI and BSC. The success of deconvolution is critical for PPI and

5 BSC analyses on event-related designed data. The different ways of dealing with deconvolutions or

6 single trial estimations may partly contribute to the different results between PPI and BSC analyses.

7 Further studies may seek to improve the deconvolution process to improve both PPI and BSC. For

8 example, more sophisticated filters could be used for deconvolution, e.g. cubature Kalman filtering

9 (Havlicek, Friston, Jan, Brazdil, \& Calhoun, 2011). In addition, applying subject-specific or region-

10 specific HRF (Pedregosa, Eickenberg, Ciuciu, Thirion, \& Gramfort, 2015) may also be helpful given the

11 large variability of HRFs (Handwerker et al., 2004). Lastly, optimizations of event-related design may

12 also improve the deconvolution process and single trial estimates, thus providing better connectivity

13 estimates for both methods (Abdulrahman \& Henson, 2016; Mumford et al., 2014; Visser et al., 2016).

\section{5. Conclusion}

16 In the current paper, we explained how PPI and BSC measure task modulated connectivity. PPI is a

17 model based approach to examine differences in effective connectivity in difference task conditions. In

18 contrast, a BSC measures correlations of observed trial-by-trial activations in a certain condition.

19 However, when comparing the differences between task conditions, BSC should in principle yield similar

20 results as shown by PPI. 


\section{Author contributions}

2 X.D. conceived the idea, performed the data analysis, and wrote the draft. All authors discussed the

3 results, and contributed to the final manuscript.

4

\section{Funding Sources}

6 This study was supported by grants from National Natural Science Foundation of China (NSFC61871420)

7 and (US) National Institute of Health (R01 AT009829; R01 DA038895).

8

\section{Compliance with Ethical Standards}

10 This study involves re-analysis of an open-access fMRI dataset. We did not use any personal identifiable

11 information in the current analysis.

12

\section{Conflict of interest}

14 The authors declare that there is no conflict of interest regarding the publication of this article. 


\section{Figure legends}

2 Figure 1 Main effects and interaction models for two experimental conditions. The main effects of two

3 conditions can be modeled as two separate regressors (A), or modeled as the differential and mean effects

4 of the two conditions (B). When modeling the interaction terms of the experimental condition with a

5 continuous variable, the same two strategies could be used as C and D. E illustrates how the interaction

6 term was changed (from D) when centering the psychological variable before calculating the interaction

7 term. Because of the different modeling strategies, the interpretations of the regressors changed.

8

Figure 2 The interaction term as a projection of the continuous (physiological) variable. A continuous variable $(\mathrm{A})$ is multiplied with a psychological variable (B or $\mathrm{E})$ to form an interaction term $(\mathrm{C}$ or $\mathrm{F})$, which can be plotted against the continuous variable itself ( $D$ or $G$ ). When the psychological variable is coded as 0 and $1(\mathrm{~B})$, the projection will result in a horizontal line $(y=0)$ during the 0 period and a $y=x$ line during the 1 period. But usually the psychological variable is centered $(\mathrm{E})$. Therefore, the projection represents $y=-0.5 \cdot x$ and $y=0.5 \cdot x$ lines during the two conditions, respectively.

Figure 3 Illustrations of "generalized" PPI and contrast PPI for three conditions. Because of the inclusion of the constant term, two psychological variables are needed to model the differences among the three conditions. For the "generalized" PPI approach, the two psychological variables are demonstrated as $\mathrm{B}$ and $\mathrm{D}$, which represent one specific condition against the other two conditions. The corresponding PPI terms were plotted against the physiological variable (A) in C and E. For the contrast PPI approach, the two psychological variables are demonstrated as $\mathrm{F}$ and $\mathrm{H}$, which represent the differential and mean effects of the last two conditions. The corresponding PPI terms were plotted against the physiological variable (A) in $\mathrm{G}$ and $\mathrm{I}$.

Figure 4 Hemodynamic response function $h(\mathrm{~A})$ and its corresponding convolution matrix $H(\mathrm{~B})$. 
1 Figure 5 Example design matrices for beta series correlation (BSC) analysis for a slow event-related

2 design (Flanker task) (A) and a fast event-related design (Stop signal task) (B). Each regressor (column)

3 except the last one represents the activation of a trial, while the last column represents the constant term.

4 The sampling time is $2 \mathrm{~s}$ for both of the designs. The intertrial intervals for both the designs were

5 randomized to optimize the estimations of hemodynamic responses. The mean intertrial intervals are $12 \mathrm{~s}$

6 for the Flanker task and $2.5 \mathrm{~s}$ for the Stop signal task, which result in 24 trials and 126 trials, respectively.

8 Figure 6 Psychophysiological interaction (PPI) and beta series correlation (BSC) results from the stop

9 signal task. The top row showed the PPI matrices using the "generalized" PPI model, where the Go

10 condition and Stop condition were modeled separately. The middle row showed correlation matrices

11 using the beta series method. The bottom row shows the scatter plots between the PPI and BSC matrices

12 of the corresponding columns. The right-side color scales of all matrices were made sure to be positive

13 and negative symmetrical, but the range was adjusted based on the values in each matrix. The left and

14 bottom color bars indicate the seven functional modules, including cerebellar, cingulo-opercular, default

15 mode, fronto-parietal, occipital, sensorimotor, and emotion modules from dark blue to dark red.

17 Figure 7 Resting-state functional connectivity matrix (mean Fisher's z) from a subset of 103 subjects (A), and its relationship with the beta series correlations (BSC) matrices of single conditions (B and C).

Figure 8 Psychophysiological interaction (PPI) results of the mean effects (A) and differential effects (B)

21 between the Stop and Go conditions using contrast PPI. C shows the relationship between the Stop - Go

22 contrasts calculated from the contrast and "generalize" PPI methods.

24 Figure 9 Mean PPI effects of the Go and Stop trials compared with the implicit baseline. A shows the 25 thresholded PPI matrix at $\mathrm{p}<0.05$ of FDR (false discovery rate) correction. Yellow represents positive 26 PPI effects, while blue represents negative effects. The color bars indicate the seven functional modules, 
1 including cerebellar, cingulo-opercular, default mode, fronto-parietal, occipital, sensorimotor, and

2 emotion modules from dark blue to dark red. B and C show the positive and negative effects on a brain

3 model using BrainNet Viewer.

4

$5 \quad$ Figure 10 Unthresholded (upper row) and thresholded (lower row) matrices of task modulated

6 connectivity between the Stop and Go conditions estimated by different methods. A p $<0.05$ of false

7 discovery rate (FDR) correction was used to threshold each matrix. The color scales of all matrices were

8 made sure to be positive and negative symmetrical. But the range was adjusted based on the values in

9 each matrix. 


\section{Reference:}

Abdulrahman, H., \& Henson, R. N. (2016). Effect of trial-to-trial variability on optimal event-related fMRI design: Implications for Beta-series correlation and multi-voxel pattern analysis. NeuroImage, 125, 756-766. http://doi.org/10.1016/j.neuroimage.2015.11.009

Biswal, B. B., Mennes, M., Zuo, X.-N., Gohel, S., Kelly, C., Smith, S. M., ... Milham, M. P. (2010). Toward discovery science of human brain function. Proceedings of the National Academy of Sciences of the United States of America, 107(10), 4734-9. http://doi.org/10.1073/pnas.0911855107

Biswal, B., Yetkin, F. Z., Haughton, V. M., \& Hyde, J. S. (1995). Functional connectivity in the motor cortex of resting human brain using echo-planar MRI. Magnetic Resonance in Medicine $\square$ : Official Journal of the Society of Magnetic Resonance in Medicine / Society of Magnetic Resonance in Medicine, 34(4), 537-41. http://doi.org/10.1002/mrm.1910340409

Cisler, J. M., Bush, K., \& Steele, J. S. (2014). A comparison of statistical methods for detecting contextmodulated functional connectivity in fMRI. NeuroImage, 84, 1042-1052. http://doi.org/10.1016/j.neuroimage.2013.09.018

Cole, M. W., Bassett, D. S., Power, J. D., Braver, T. S., \& Petersen, S. E. (2014). Intrinsic and taskevoked network architectures of the human brain. Neuron, 83, 238-251. http://doi.org/10.1016/j.neuron.2014.05.014

Dale, A. M. (1999). Optimal experimental design for event-related fMRI. Human Brain Mapping, 8(2-3), 109-114. http://doi.org/10.1002/(SICI)1097-0193(1999)8:2/3<109::AID-HBM7>3.0.CO;2-W

Di, X., \& Biswal, B. B. (2014). Identifying the default mode network structure using dynamic causal modeling on resting-state functional magnetic resonance imaging. NeuroImage, 86(null), 53-9. http://doi.org/10.1016/j.neuroimage.2013.07.071

Di, X., \& Biswal, B. B. (2017). Psychophysiological Interactions in a Visual Checkerboard Task: Reproducibility, Reliability, and the Effects of Deconvolution. Front Neurosci, 1-36. http://doi.org/10.3389/fnins.2017.00573

Di, X., \& Biswal, B. B. (2019). Toward Task Connectomics: Examining Whole-Brain Task Modulated 
Connectivity in Different Task Domains. Cerebral Cortex, 29(4), 1572-1583. http://doi.org/10.1093/cercor/bhy055

Di, X., Reynolds, R. C., \& Biswal, B. B. (2017). Imperfect (de)convolution may introduce spurious psychophysiological interactions and how to avoid it. Human Brain Mapping, 38(4), 1723-1740. http://doi.org/10.1002/hbm.23413

Duff, E. P., Makin, T., Cottaar, M., Smith, S. M., \& Woolrich, M. W. (2018). Disambiguating brain

Friston, K. J. (2011). Functional and effective connectivity: a review. Brain Connectivity, 1(1), 13-36.

Friston, K. J. (1994). Functional and effective connectivity in neuroimaging: A synthesis. Human Brain Mapping, 2(1-2), 56-78. http://doi.org/10.1002/hbm.460020107

Friston, K. J., Buechel, C., Fink, G. R., Morris, J., Rolls, E., \& Dolan, R. J. (1997). Psychophysiological

Friston, K. J., Fletcher, P., Josephs, O., Holmes, A., Rugg, M. D., \& Turner, R. (1998). Event-related and modulatory interactions in neuroimaging. NeuroImage, 6(3), 218-29. Retrieved from http://www.ncbi.nlm.nih.gov/pubmed/9344826 fMRI: characterizing differential responses. NeuroImage, 7(1), 30-40. http://doi.org/10.1006/nimg.1997.0306

Gitelman, D. R., Penny, W. D., Ashburner, J., \& Friston, K. J. (2003). Modeling regional and psychophysiologic interactions in fMRI: the importance of hemodynamic deconvolution. NeuroImage, 19(1), 200-7. Retrieved from http://www.ncbi.nlm.nih.gov/pubmed/12781739

Handwerker, D. A., Ollinger, J. M., \& D’Esposito, M. (2004). Variation of BOLD hemodynamic responses across subjects and brain regions and their effects on statistical analyses. NeuroImage, 21(4), 1639-1651. http://doi.org/10.1016/j.neuroimage.2003.11.029

Havlicek, M., Friston, K. J., Jan, J., Brazdil, M., \& Calhoun, V. D. (2011). Dynamic modeling of neuronal responses in fMRI using cubature Kalman filtering. NeuroImage, 56(4), 2109-2128. 
Hillman, E. M. C. (2014). Coupling Mechanism and Significance of the BOLD Signal: A Status Report. Annual Review of Neuroscience, 37(1), 161-181. http://doi.org/10.1146/annurev-neuro-071013014111

Honey, C. J., Sporns, O., Cammoun, L., Gigandet, X., Thiran, J. P., Meuli, R., \& Hagmann, P. (2009). Predicting human resting-state functional connectivity from structural connectivity. Proceedings of the National Academy of Sciences of the United States of America, 106(6), 2035-40. http://doi.org/10.1073/pnas.0811168106

Kelly, A. M. C., Uddin, L. Q., Biswal, B. B., Castellanos, F. X., \& Milham, M. P. (2008). Competition between functional brain networks mediates behavioral variability. NeuroImage, 39(1), 527-37. http://doi.org/10.1016/j.neuroimage.2007.08.008

McLaren, D. G., Ries, M. L., Xu, G., \& Johnson, S. C. (2012). A generalized form of context-dependent psychophysiological interactions (gPPI): A comparison to standard approaches. NeuroImage, 61(4), 1277-1286. http://doi.org/10.1016/j.neuroimage.2012.03.068

Mumford, J. A., Davis, T., \& Poldrack, R. A. (2014). The impact of study design on pattern estimation for single-trial multivariate pattern analysis. NeuroImage, 103, 130-138. http://doi.org/10.1016/j.neuroimage.2014.09.026

Mumford, J. A., Turner, B. O., Ashby, F. G., \& Poldrack, R. A. (2012). Deconvolving BOLD activation in event-related designs for multivoxel pattern classification analyses. NeuroImage, 59(3), 26362643. http://doi.org/10.1016/j.neuroimage.2011.08.076

O’Reilly, J. X., Woolrich, M. W., Behrens, T. E. J., Smith, S. M., \& Johansen-Berg, H. (2012). Tools of the Trade: Psychophysiological Interactions and Functional Connectivity. Social Cognitive and Affective Neuroscience, nss055-. http://doi.org/10.1093/scan/nss055

Park, H.-J., \& Friston, K. (2013). Structural and Functional Brain Networks: From Connections to Cognition. Science, 342(6158), 1238411-1238411. http://doi.org/10.1126/science.1238411

Pedregosa, F., Eickenberg, M., Ciuciu, P., Thirion, B., \& Gramfort, A. (2015). Data-driven HRF 
estimation for encoding and decoding models. NeuroImage, 104, 209-220. http://doi.org/10.1016/j.neuroimage.2014.09.060

Poldrack, R. A., Congdon, E., Triplett, W., Gorgolewski, K. J., Karlsgodt, K. H., Mumford, J. A., ... Bilder, R. M. (2016). A phenome-wide examination of neural and cognitive function. Scientific Data, 3, 160110. http://doi.org/10.1038/sdata.2016.110

Power, J. D., Barnes, K. A., Snyder, A. Z., Schlaggar, B. L., \& Petersen, S. E. (2012). Spurious but

Rowe, J. B., Stephan, K. E., Friston, K., Frackowiak, R. S. J., \& Passingham, R. E. (2005). The prefrontal cortex shows context-specific changes in effective connectivity to motor or visual cortex during the

Sivakolundu, D. K., West, K. L., Maruthy, G. B., Zuppichini, M., Turner, M. P., Abdelkarim, D., ... selection of action or colour. Cerebral Cortex (New York, N.Y. $\square: 1991), 15(1), 85-95$. http://doi.org/10.1093/cercor/bhh111

(7)
Rypma, B. (2019). Reduced arterial compliance along the cerebrovascular tree predicts cognitive slowing in multiple sclerosis: Evidence for a neurovascular uncoupling hypothesis. Multiple Sclerosis Journal, 135245851986660. http://doi.org/10.1177/1352458519866605

Van Dijk, K. R. A., Sabuncu, M. R., \& Buckner, R. L. (2012). The influence of head motion on intrinsic functional connectivity MRI. NeuroImage, 59(1), 431-438. http://doi.org/10.1016/j.neuroimage.2011.07.044

Visser, R. M., de Haan, M. I. C., Beemsterboer, T., Haver, P., Kindt, M., \& Scholte, H. S. (2016). Quantifying learning-dependent changes in the brain: Single-trial multivoxel pattern analysis requires slow event-related fMRI. Psychophysiology, 53(8), 1117-1127. http://doi.org/10.1111/psyp.12665 
1 Weissenbacher, A., Kasess, C., Gerstl, F., Lanzenberger, R., Moser, E., \& Windischberger, C. (2009).

2 Correlations and anticorrelations in resting-state functional connectivity MRI: a quantitative

3 comparison of preprocessing strategies. NeuroImage, 47(4), 1408-16.

$4 \quad$ http://doi.org/10.1016/j.neuroimage.2009.05.005

5 Xia, M., Wang, J., \& He, Y. (2013). BrainNet Viewer: a network visualization tool for human brain

6 connectomics. PloS One, 8(7), e68910. http://doi.org/10.1371/journal.pone.0068910 

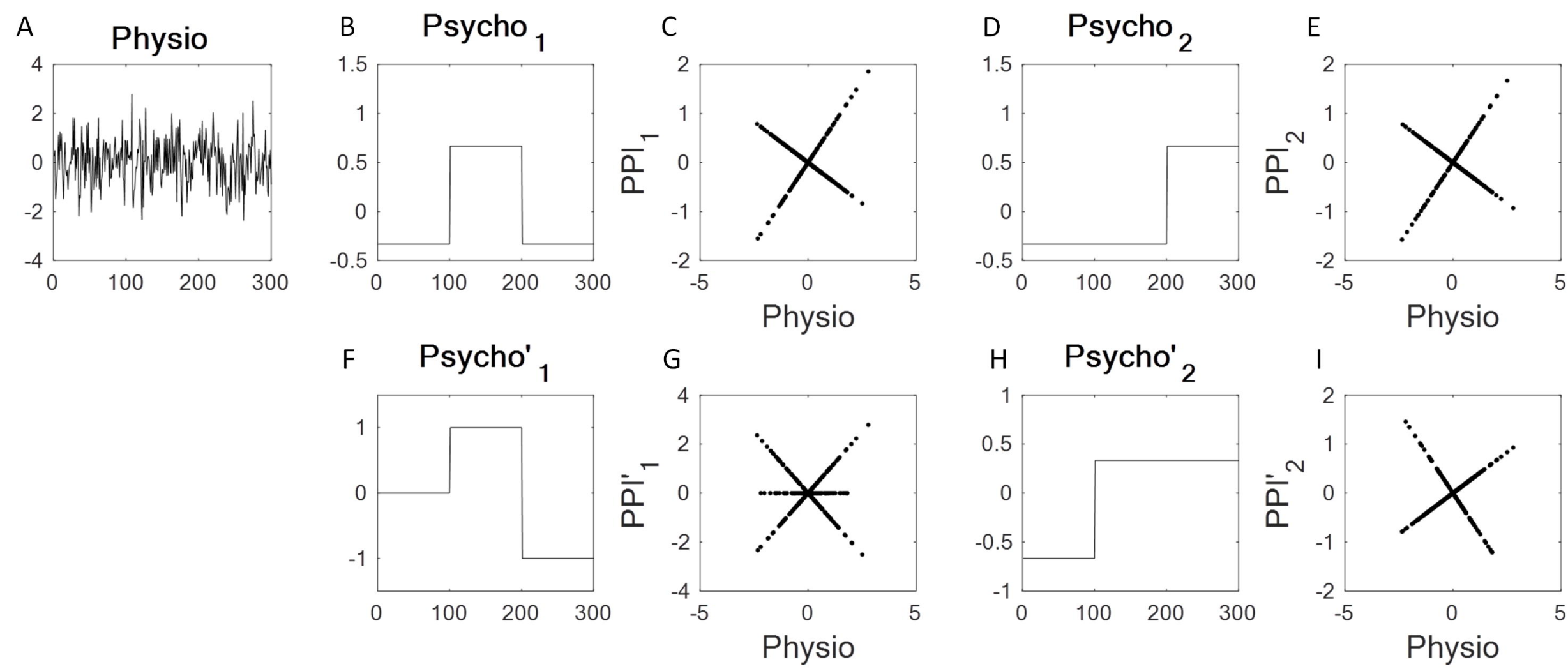

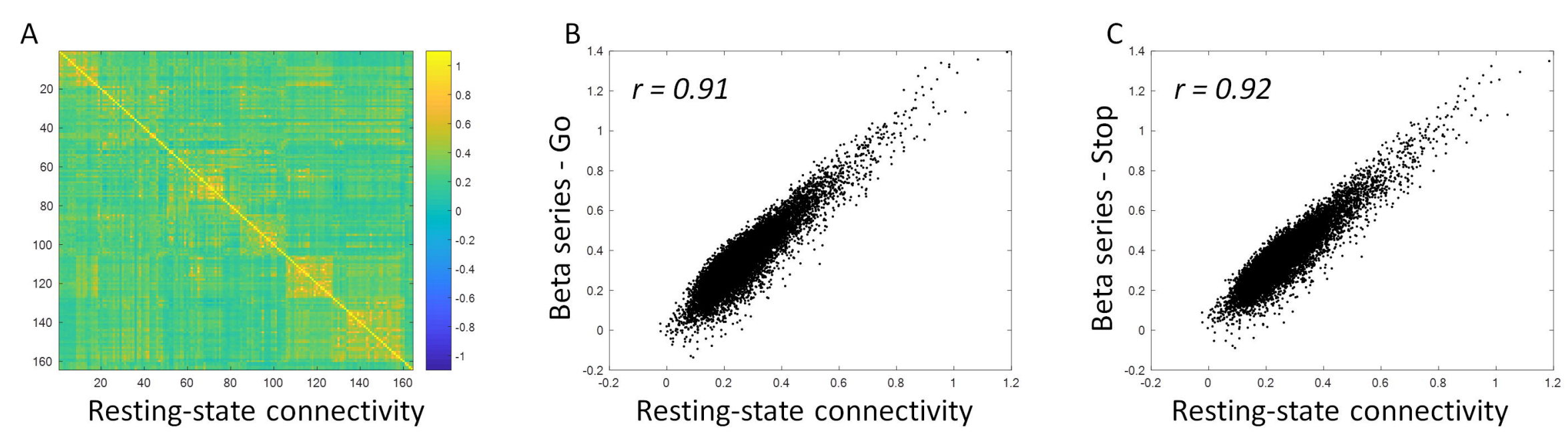


$$
\text { A } \quad(G o+\text { Stop })-\text { Rest }
$$

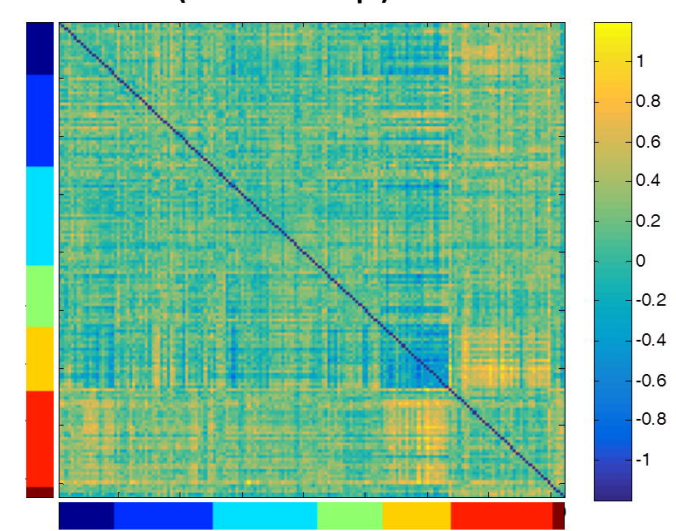

Stop - Go (Rest $=0)$

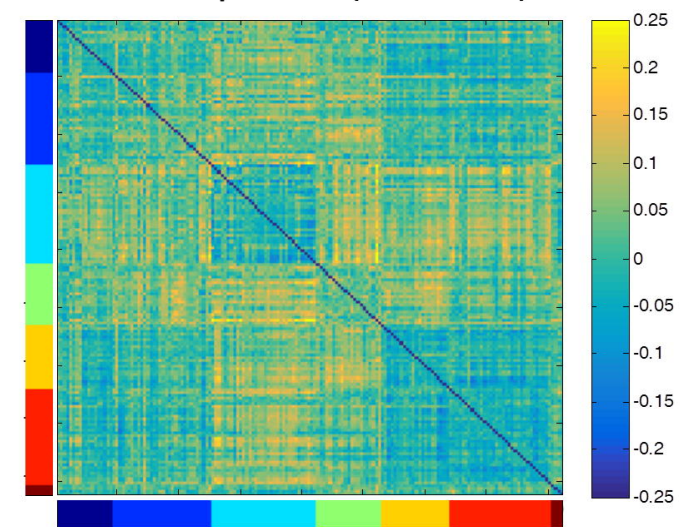

C

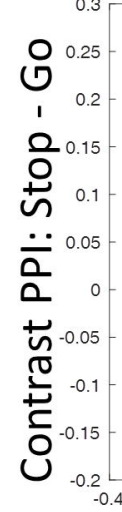

$$
r=1
$$
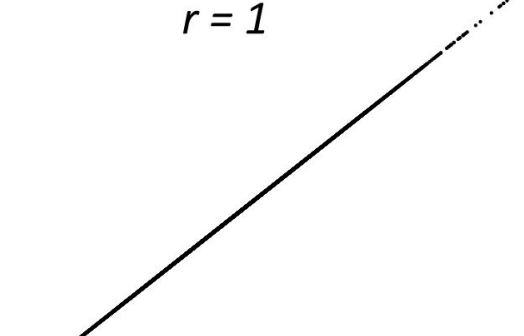
A

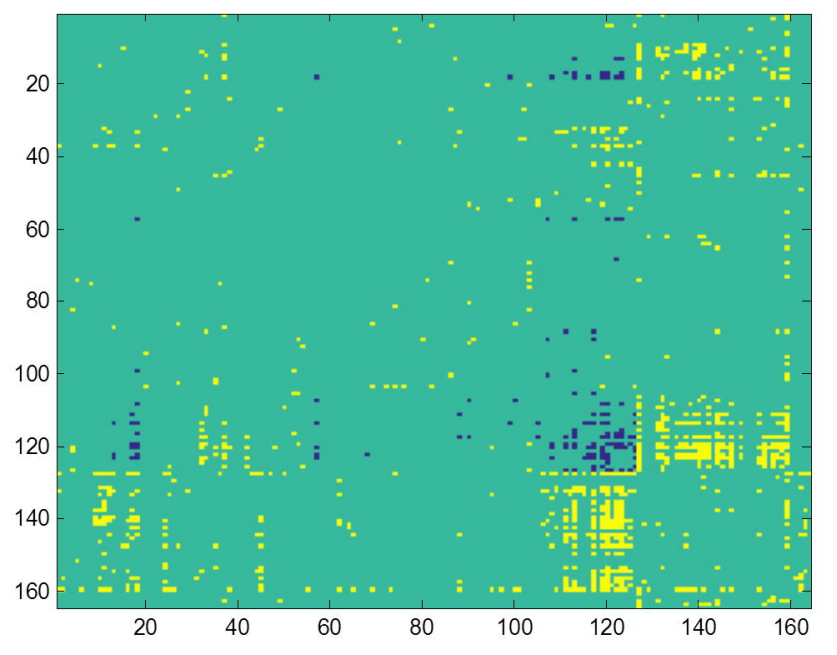

B

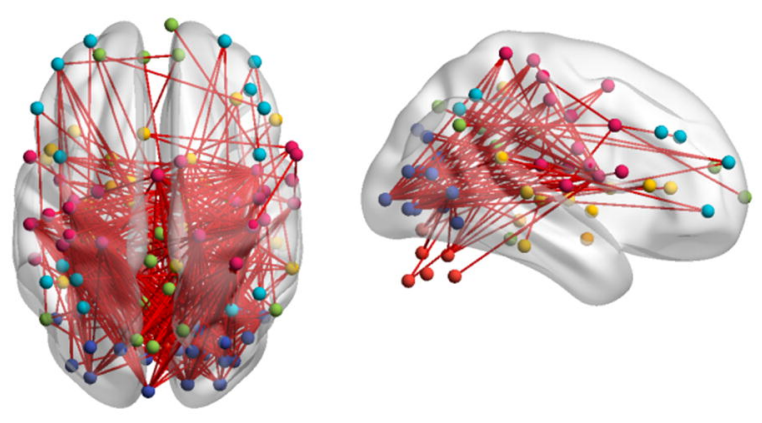

C

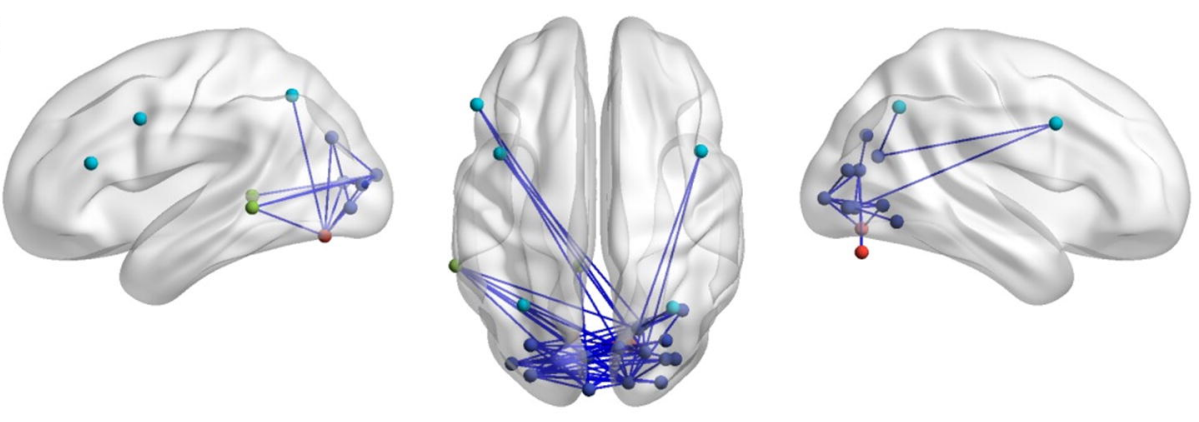


PPI

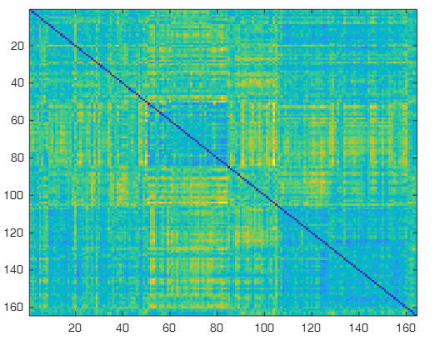

BSC: Spearman
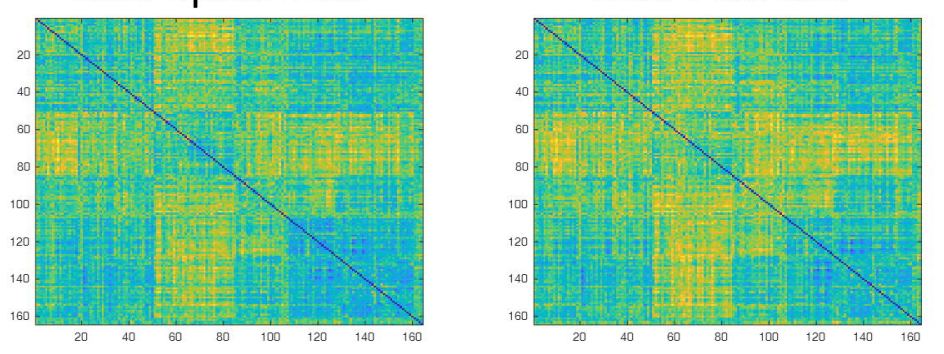

BSC: Covariance

BSC: Interaction
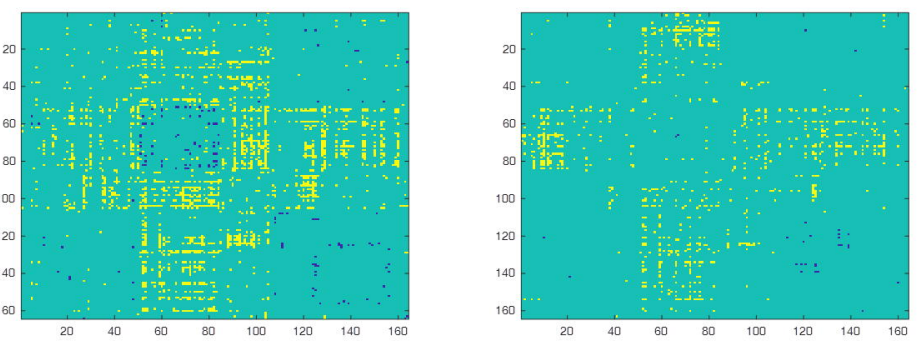
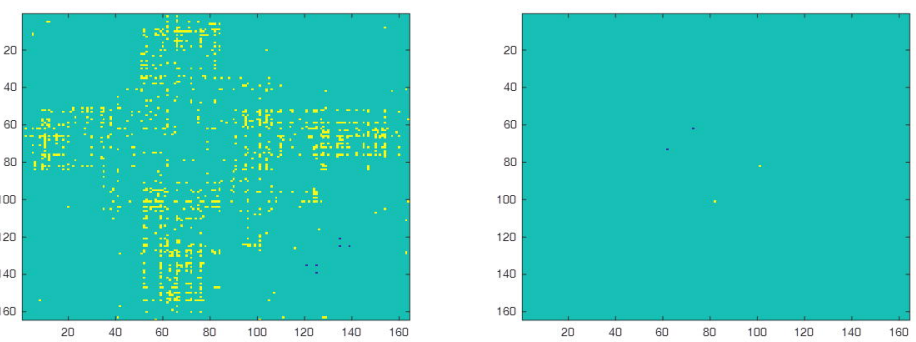

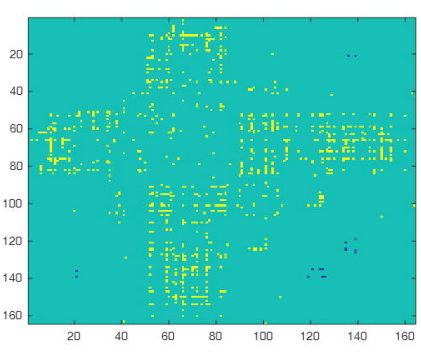

